JLAB-THY-98-39

hep-ph/9810254

\title{
Isospin violation and the proton's neutral weak magnetic form factor
}

\author{
Randy Lewis ${ }^{1,2}$ and Nader Mobed $^{2}$ \\ ${ }^{1}$ Jefferson Lab, 12000 Jefferson Avenue, Newport News, VA, U.S.A. 23606 \\ ${ }^{2}$ Department of Physics, University of Regina, Regina, SK, Canada S4S 0A2
}

(October 1998)

\begin{abstract}
The effects of isospin violation on the neutral weak magnetic form factor of the proton are studied using two-flavour chiral perturbation theory. The first nonzero contributions appear at $O\left(p^{4}\right)$ in the small-momentum expansion, and the $O\left(p^{5}\right)$ corrections are also calculated. The leading contributions from an explicit $\Delta(1232)$ isomultiplet are included as well. At such a high order in the chiral expansion, one might have expected a large number of unknown parameters to contribute. However, it is found that no unknown parameters can appear within loop diagrams, and a single tree-level counterterm at $O\left(p^{4}\right)$ is sufficient to absorb all divergences. The momentum dependence of the neutral weak magnetic form factor is not affected by this counterterm.
\end{abstract}




\section{INTRODUCTION}

The first measurement of the proton's neutral weak magnetic form factor has been reported recently by the SAMPLE Collaboration at MIT/Bates [1]; subsequently, a series of precision experiments has gotten underway at the Jefferson Lab[2]. Assuming only two quark flavours with exact isospin symmetry and neglecting electroweak radiative corrections, the weak form factor can be expressed in terms of the familiar electromagnetic form factors as follows:

$$
G_{M}^{p, Z}\left(q^{2}\right)=\frac{1}{4}\left[G_{M}^{p}\left(q^{2}\right)-G_{M}^{n}\left(q^{2}\right)\right]-G_{M}^{p}\left(q^{2}\right) \sin ^{2} \theta_{W} .
$$

Electroweak radiative corrections have been discussed in Ref. [3].

There is presently a great deal of interest in determining the contribution due to strange quarks [4], which simply appears as a new term added to the right-hand side of Eq. (1). It is important to notice that isospin-violating effects also appear as a new term on the right-hand side, even in the absence of strange quarks. There have been some attempts to estimate the isospin-violating effects by using constituent quark models [5, 6, 7] and a light-cone meson-baryon fluctuation model [8].

In the present work, the effects of isospin violation are studied using two-flavour heavy baryon chiral perturbation theory (HBChPT) [9, 10], which is nicely suited to the task. At small momentum transfer, HBChPT is a systematic expansion in small parameters with dynamics arising from the propagation of pions and photons in the presence of a single baryon. The remaining short-distance physics can only appear as low-energy constants (i.e. parameters in the HBChPT Lagrangian). The spontaneously-broken chiral symmetry of QCD is respected in HBChPT by construction, and the explicit breaking due to current quark masses can also be included in a systematic way.

Isospin violation occurs in nature through electromagnetic as well as strong interactions, so it is necessary to include the effects of virtual photons in HBChPT. The required Lagrangian has been constructed by Meissner and Steininger 11.

In principle, three-flavour HBChPT can be used to explicitly include strangeness in the meson-cloud contribution to the neutral weak form factors, and some work in this direction has recently been reported. [12] However, the chiral expansion is not as wellbehaved for strange quarks as it is for up and down quarks, and since the present work is only concerned with isospin-violating effects it is preferable to work with two-flavour 


\section{HBChPT.}

The neutral weak vector form factors of the nucleons are defined here in the notation of Dmitrašinović and Pollock[5],

$$
\begin{aligned}
\left\langle N(\vec{p}+\vec{q})\left|\frac{1}{2}\left(\bar{u} \gamma_{\mu} u-\bar{d} \gamma_{\mu} d\right)\right| N(\vec{p})\right\rangle= & \bar{u}(\vec{p}+\vec{q})\left[\frac{1}{2}\left({ }^{u-d} F_{1}^{p+n} \pm{ }^{u-d} F_{1}^{p-n}\right) \gamma_{\mu}\right. \\
& \left.+\frac{1}{2}\left({ }^{u-d} F_{2}^{p+n} \pm^{u-d} F_{2}^{p-n}\right) \frac{i \sigma_{\mu \nu} q^{\nu}}{2 M_{N}}\right] u(\vec{p}), \\
\left\langle N(\vec{p}+\vec{q})\left|\frac{1}{6}\left(\bar{u} \gamma_{\mu} u+\bar{d} \gamma_{\mu} d\right)\right| N(\vec{p})\right\rangle= & \bar{u}(\vec{p}+\vec{q})\left[\frac{1}{2}\left({ }^{u+d} F_{1}^{p+n} \pm{ }^{u+d} F_{1}^{p-n}\right) \gamma_{\mu}\right. \\
& \left.+\frac{1}{2}\left({ }^{u+d} F_{2}^{p+n} \pm{ }^{u+d} F_{2}^{p-n}\right) \frac{i \sigma_{\mu \nu} q^{\nu}}{2 M_{N}}\right] u(\vec{p}),
\end{aligned}
$$

with $M_{N}$ denoting a nucleon mass. Walecka-Sachs form factors are defined by

$$
\begin{aligned}
{ }^{i} G_{E}^{j}\left(q^{2}\right) & ={ }^{i} F_{1}^{j}\left(q^{2}\right)+\frac{q^{2}}{4 M_{N}^{2}}{ }^{i} F_{2}^{j}\left(q^{2}\right), \\
{ }^{i} G_{M}^{j}\left(q^{2}\right) & ={ }^{i} F_{1}^{j}\left(q^{2}\right)+{ }^{i} F_{2}^{j}\left(q^{2}\right),
\end{aligned}
$$

where $i=u \pm d$ and $j=p \pm n$. When isospin conservation is not enforced, Eq. (11) generalizes to

$$
G_{M}^{p, Z}\left(q^{2}\right)=\frac{1}{4}\left[G_{M}^{p}\left(q^{2}\right)-G_{M}^{n}\left(q^{2}\right)\right]-G_{M}^{p}\left(q^{2}\right) \sin ^{2} \theta_{W}-\frac{1}{4} G_{M}^{u, d}\left(q^{2}\right),
$$

where

$$
G_{M}^{u, d}\left(q^{2}\right) \equiv{ }^{u+d} G_{M}^{p-n}\left(q^{2}\right)-{ }^{u-d} G_{M}^{p+n}\left(q^{2}\right)
$$

is the isospin-violating term.

In section 2, it is shown that isospin-violating contributions do not appear up to and including $O\left(p^{3}\right)$ in HBChPT. Section 3 presents and discusses the $O\left(p^{4}\right)$ calculation. At this order, the isospin-violating ${ }^{i} F_{1}^{j}$ form factors contain no unknown parameters. Each ${ }^{i} F_{2}^{j}$ requires a single counterterm, but the momentum dependence is not affected by the counterterm.

In section 4 , the calculation of $G_{M}^{u, d}\left(q^{2}\right)$ is extended to next-to-leading order, $O\left(p^{5}\right)$. In principle, a large number of low-energy constants could appear, some within loop diagrams and others as tree-level counterterms, but it is shown that the loop integrals are finite and no new low-energy constants appear at this order. The ratio of next-toleading versus leading order contributions provides some indication of the behaviour of 
the HBChPT expansion. For the derivative of $G_{M}^{u, d}\left(q^{2}\right)$ at $q^{2}=0$, this ratio is close to $1 / 2$.

Section 5 evaluates and discusses the contributions to $G_{M}^{u, d}\left(q^{2}\right)$ that arise when the $\Delta(1232)$ is included explicitly in the chiral Lagrangian. Section 6 offers a determination of the pion-cloud contribution to the sole remaining parameter in $G_{M}^{u, d}\left(q^{2}\right)$, and then summarizes the complete HBChPT result for $G_{M}^{u, d}\left(q^{2}\right)$.

\section{NO CONTRIBUTION UP TO $O\left(p^{3}\right)$}

The Lagrangian of HBChPT is written in the form

$$
\mathcal{L}_{\pi N}=\mathcal{L}_{\pi N}^{(1)}+\mathcal{L}_{\pi N}^{(2)}+\mathcal{L}_{\pi N}^{(3)}+\mathcal{L}_{\pi N}^{(4)}+\mathcal{L}_{\pi N}^{(5)}+\ldots
$$

where the superscripts denote powers in the "momentum" expansion, which is actually a combined expansion in various small quantities. A covariant derivative counts as one power (therefore the field strength of an external current counts as two powers) and a current quark mass counts as two powers (recall that $m_{\pi}^{2} \sim m_{q}$ ). These dimensionful quantities are small relative to both the chiral scale and the nucleon masses, $4 \pi F_{\pi} \approx M_{p} \approx M_{n}$. Eq. (8) represents an expansion in the inverse nucleon masses as well as the chiral expansion. Furthermore, the effects of virtual photons can be organized according to an expansion in the electromagnetic coupling, and it is convenient to use $O(e) \sim O(p)$ [11], which allows the virtual photon effects to also be incorporated into the generalized momentum expansion of Eq. (8). In the present work, "O( $\left.p^{n}\right)$ " is used to denote $n$ powers of any of these small quantities.

The lowest-order Lagrangian is

$$
\mathcal{L}_{\pi N}^{(1)}=\bar{N}_{v}\left(i v \cdot \nabla+g_{A} S \cdot u\right) N_{v}
$$

where

$$
\begin{aligned}
N_{v}(x) & =\exp \left[i M_{0} v \cdot x\right] \frac{1}{2}(1+\psi) \psi(x), \\
S_{\mu} & =\frac{i}{2} \gamma_{5} \sigma_{\mu \nu} v^{\nu} \\
u_{\mu} & =i u^{\dagger}\left(\partial_{\mu}-i r_{\mu}\right) u-i u\left(\partial_{\mu}-i \ell_{\mu}\right) u^{\dagger}, \\
\nabla_{\mu} & =\partial_{\mu}+\Gamma_{\mu}-i v_{\mu}^{(s)},
\end{aligned}
$$




$$
\Gamma_{\mu}=\frac{1}{2}\left[u^{\dagger}\left(\partial_{\mu}-i r_{\mu}\right) u+u\left(\partial_{\mu}-i \ell_{\mu}\right) u^{\dagger}\right]
$$

and $M_{0}$ is the lowest-order nucleon mass. External vector and axial vector fields are included via $r_{\mu}=V_{\mu}+A_{\mu}$ and $\ell_{\mu}=V_{\mu}-A_{\mu}$, and $u$ is a nonlinear representation of the pion fields, for example

$$
u=\exp \left[\frac{i}{2 F}\left(\begin{array}{cc}
\pi^{0} & \sqrt{2} \pi^{+} \\
\sqrt{2} \pi^{-} & -\pi^{0}
\end{array}\right)\right] .
$$

The parameter $F$ corresponds to the pion decay constant in the chiral limit (normalized according to $F_{\pi} \approx 93 \mathrm{MeV}$ ).

The following relations will also be useful:

$$
\begin{aligned}
S \cdot v & =0 \\
\left\{S_{\mu}, S_{\nu}\right\} & =\frac{1}{2}\left(v_{\mu} v_{\nu}-g_{\mu \nu}\right) \\
{\left[S_{\mu}, S_{\nu}\right] } & =i \epsilon_{\mu \nu \rho \omega} v^{\rho} S^{\omega}
\end{aligned}
$$

The relativistic currents required for this work can be re-expressed as a $1 / M_{N}$ expansion between HBChPT spinors. In the rest frame of the initial nucleon, one finds

$$
\begin{aligned}
\bar{\psi}(\vec{p}+\vec{q}) \gamma_{\mu} \psi(\vec{p}) & =\bar{N}_{v}\left[v_{\mu}+\frac{q_{\mu}}{2 M_{N}}+\frac{1}{M_{N}} i \epsilon_{\mu \nu \rho \sigma} q^{\nu} v^{\rho} S^{\sigma}+O\left(\frac{1}{M_{N}^{2}}\right)\right] N_{v}, \\
\bar{\psi}(\vec{p}+\vec{q}) \frac{i \sigma_{\mu \nu} q^{\nu}}{2 M_{N}} \psi(\vec{p}) & =\bar{N}_{v}\left[\frac{1}{M_{N}} i \epsilon_{\mu \nu \rho \omega} q^{\nu} v^{\rho} S^{\omega}+\frac{v_{\mu} q^{2}}{4 M_{N}^{2}}+O\left(\frac{1}{M_{N}^{3}}\right)\right] N_{v},
\end{aligned}
$$

where $v_{\mu}=(1,0,0,0), q_{\mu}$ is the 4-momentum of the incoming vector current and $M_{N}$ is the physical mass of the nucleon.

It is a simple matter to determine from $\mathcal{L}_{\pi N}^{(1)}$ the tree-level coupling of an external neutral vector field to a nucleon. The result is

$$
\begin{aligned}
& { }^{u-d} F_{1}^{p-n}={ }^{u+d} F_{1}^{p+n}=1, \\
& { }^{u-d} F_{1}^{p+n}={ }^{u+d} F_{1}^{p-n}={ }^{i} F_{2}^{j}=0, \text { for all } i, j .
\end{aligned}
$$

No contributions to the ${ }^{i} F_{2}^{j}$ form factors can appear at this order due to the explicit factor of $q / M_{N}$ in Eqs. (2) and (3). Also, loop graphs are forbidden at $O(p)$ by the standard power counting of HBChPT, which assures that one-loop diagrams constructed from $\mathcal{L}_{\pi N}^{(1)}$ begin at $O\left(p^{3}\right)$. 
The isospin-violating form factors must contain $m_{u}-m_{d}$ or a virtual photon (with the associated factor of $e^{2}$ ), and are therefore suppressed by at least two powers relative to the isospin-conserving ones. This guarantees that ${ }^{u-d} F_{2}^{p+n}$ and ${ }^{u+d} F_{2}^{p-n}$ must remain zero until $O\left(p^{4}\right)$. One might expect ${ }^{u-d} F_{1}^{p+n}$ and ${ }^{u+d} F_{1}^{p-n}$ to be nonzero at $O\left(p^{3}\right)$, but in fact they also remain zero until $O\left(p^{4}\right)$ due to Noether's theorem and the fact that the vector currents $\bar{u} \gamma_{\mu} u$ and $\bar{d} \gamma_{\mu} d$ are each conserved in QCD and QED (recall that weak radiative corrections are being neglected). Noether's theorem requires that the ${ }^{i} F_{1}^{j}$ form factors of Eqs. (21) and (22) do not get renormalized at $q^{2}=0$, but explicit factors of $q^{2}$ cannot appear until $O\left(p^{4}\right)$ in loop diagrams or $O\left(p^{5}\right)$ at tree level.

Finally then, it is concluded that all isospin-violating form factors remain zero up to and including $O\left(p^{3}\right)$. It is instructive to verify this fact by a direct calculation using the chiral Lagrangian which has been written down in its entirety at this order. [13], 14, [1] Following the notation of Ref. [13] where field redefinitions have been used to remove "equation of motion" terms from the Lagrangian, the $O\left(p^{2}\right)$ terms which affect the neutral vector form factors are

$$
\delta \mathcal{L}_{\pi N}^{(2)}=\bar{N}_{v}\left[-\frac{\nabla \cdot \nabla}{2 M_{0}}+\frac{1}{M_{0}} \epsilon^{\mu \nu \rho \sigma} v_{\rho} S_{\sigma}\left(a_{6} f_{+\mu \nu}+a_{7} v_{\mu \nu}^{(s)}\right)\right] N_{v},
$$

where

$$
\begin{aligned}
f_{+\mu \nu} & =u\left(\partial_{\mu} \ell_{\nu}-\partial_{\nu} \ell_{\mu}-i\left[\ell_{\mu}, \ell_{\nu}\right]\right) u^{\dagger}+u^{\dagger}\left(\partial_{\mu} r_{\nu}-\partial_{\nu} r_{\mu}-i\left[r_{\mu}, r_{\nu}\right]\right) u \\
v_{\mu \nu}^{(s)} & =\partial_{\mu} v_{\nu}^{(s)}-\partial_{\nu} v_{\mu}^{(s)} .
\end{aligned}
$$

Adding these terms to the lowest-order Lagrangian of Eq. (9) leads to the following coupling of an isoscalar vector current to a proton:

$$
\bar{N}_{v}\left[i v_{\mu}+\frac{i}{2 M_{0}}(2 k+q)_{\mu}+\frac{2 i a_{7}}{M_{0}} i \epsilon_{\mu \nu \rho \sigma} q^{\nu} v^{\rho} S^{\sigma}\right] N_{v} .
$$

According to Eq. (23), the expression for an isovector current is obtained by the replacement $a_{7} \rightarrow 2 a_{6} . q_{\mu}$ is the incoming 4-momentum of the vector current, and the 4-vectors $v_{\mu}$ and $k_{\mu}$ are defined by

$$
p_{\mu}=M_{0} v_{\mu}+k_{\mu},
$$

where $p_{\mu}$ is the 4-momentum of the incoming nucleon. 
One is free to work in the rest frame of the initial nucleon, $p_{\mu}=\left(M_{N}, 0,0,0\right)$, and to choose $v_{\mu}=(1,0,0,0)$. For on-shell nucleons, these choices imply

$$
\begin{aligned}
k_{\mu} & =\left(M_{N}-M_{0}\right) v_{\mu} \\
v \cdot q & =\frac{-q^{2}}{2 M_{N}}
\end{aligned}
$$

and lead to

$$
\begin{aligned}
& { }^{u+d} F_{1}^{p-n}={ }^{u-d} F_{1}^{p+n}=-\frac{\left(M_{n}-M_{p}\right)}{M_{0}}, \\
& { }^{u+d} F_{2}^{p-n}={ }^{u-d} F_{2}^{p+n}=0 .
\end{aligned}
$$

Recalling that the neutron-proton mass difference is $O\left(1 / M_{0}\right)[13$, 15], one concludes that the nonzero result of Eq. (30) is suppressed by two powers of $1 / M_{0}$ relative to the leading isospin-conserving result. In other words Eq. (30) contributes at $O\left(p^{3}\right)$, and therefore the isospin-violating form factors all vanish up to $O\left(p^{2}\right)$, as expected.

When the calculation is extended to $O\left(p^{3}\right)$, Eq. (30) is not the only contribution. $\mathcal{L}_{\pi N}^{(3)}$, which includes virtual photons as well as strong interactions, contains approximately 40 new parameters. [13, 14, 11] However, none of these parameters can contribute to the isospin-violating form factors at $O\left(p^{3}\right)$, and the only terms that do contribute are

$$
\delta \mathcal{L}_{\pi N}^{(3)}=\frac{1}{2 M_{0}^{2}} \bar{N}_{v}\left(\left[\left(a_{6}-\frac{1}{8}\right) f_{+\mu \nu}+\left(a_{7}-\frac{1}{4}\right) v_{\mu \nu}^{(s)}\right] \epsilon^{\mu \nu \rho \sigma} S_{\sigma} i \nabla_{\rho}+\text { h.c. }\right) N_{v} .
$$

A simple calculation shows that these terms do not affect ${ }^{i} F_{1}^{j}$, and their contributions to ${ }^{i} F_{2}^{j}$ are effectively $O\left(p^{4}\right)$, so they are negligible in an $O\left(p^{3}\right)$ calculation.

In a general HBChPT calculation, one-loop diagrams built from $\mathcal{L}_{\pi N}^{(1)}$ interactions can contribute at $O\left(p^{3}\right)$, but none exist which can contribute to the isospin-violating form factors of interest here. However, there is a contribution from wave function renormalization 15, 16],

$$
Z_{p}-Z_{n}=\frac{M_{n}-M_{p}}{M_{0}}+O\left(\frac{1}{M_{0}^{3}}\right),
$$

and it precisely cancels the $O\left(p^{3}\right)$ effect found in Eq. (30). All $O\left(p^{3}\right)$ effects have now been discussed, so the isospin-violating form factors do indeed remain zero at this order.

It is easy to verify that the final results of this section remain unchanged if one does not employ the field redefinitions of Ref. [13, even though unphysical intermediate steps 
may differ. For example, the wave function renormalization constant at $O\left(p^{3}\right)$ becomes independent of the nucleon mass when the field redefinitions are not used, so $Z_{p}=Z_{n}$, but this change is exactly compensated by the extra equation-of-motion term that would be present in $\mathcal{L}_{\pi N}^{(2)}[13$,

$$
\mathcal{L}_{\pi N}^{(2)} \rightarrow \mathcal{L}_{\pi N}^{(2)}+\frac{1}{2 M_{0}} \bar{N}_{v}(v \cdot \nabla)^{2} N_{v}
$$

\section{LEADING ORDER, $O\left(p^{4}\right)$}

Having verified explicitly that the isospin-violating neutral weak vector form factors are exactly zero up to $O\left(p^{3}\right)$, the calculation will now be extended to $O\left(p^{4}\right)$ where a nonzero result does exist. The complete Lagrangian $\mathcal{L}_{\pi N}^{(4)}$ has not been written in the literature (but an effort is underway: the counterterms required for renormalization have recently been listed[[17]). However, the contribution of $\mathcal{L}_{\pi N}^{(4)}$ to the present study is a simple constant and the full Lagrangian need not be constructed here. Of greater interest are the $q^{2}$-dependent $O\left(p^{4}\right)$ effects which come from loop diagrams constructed using $\mathcal{L}_{\pi N}^{(1)}+\mathcal{L}_{\pi N}^{(2)}$.

All of the pion-loop diagrams which contribute to the isospin-violating form factors at $O\left(p^{4}\right)$ are shown in Fig. 11. (Diagrams with virtual photon loops will be discussed later in this section.) Each of them will be evaluated in the rest frame of the initial nucleon with the "velocity" parameter fixed to $v_{\mu}=(1,0,0,0)$. Final results for the form factors do not depend on these choices.

The pion propagator and the vector-pion couplings are obtained from the lowest-order chiral Lagrangian for mesons,

$$
\begin{aligned}
\mathcal{L}_{\pi \pi}^{(2)}= & \frac{F^{2}}{4} \operatorname{Tr}\left[D_{\mu} U^{\dagger} D^{\mu} U+2 B\left(\begin{array}{cc}
m_{u} & 0 \\
0 & m_{d}
\end{array}\right)\left(U+U^{\dagger}\right)\right. \\
& \left.+e^{2} C\left(\begin{array}{cc}
2 / 3 & 0 \\
0 & -1 / 3
\end{array}\right) U\left(\begin{array}{cc}
2 / 3 & 0 \\
0 & -1 / 3
\end{array}\right) U^{\dagger}\right]
\end{aligned}
$$

where $U \equiv u^{2}$ and

$$
D_{\mu} U=\partial_{\mu} U-i r_{\mu} U+i U \ell_{\mu}
$$

Notice that the physical pion masses are nonzero for two reasons: current quark mass effects (the parameter $B$ in $\mathcal{L}_{\pi \pi}^{(2)}$ ) and electromagnetic effects (the parameter $C$ in $\mathcal{L}_{\pi \pi}^{(2)}$ ). 


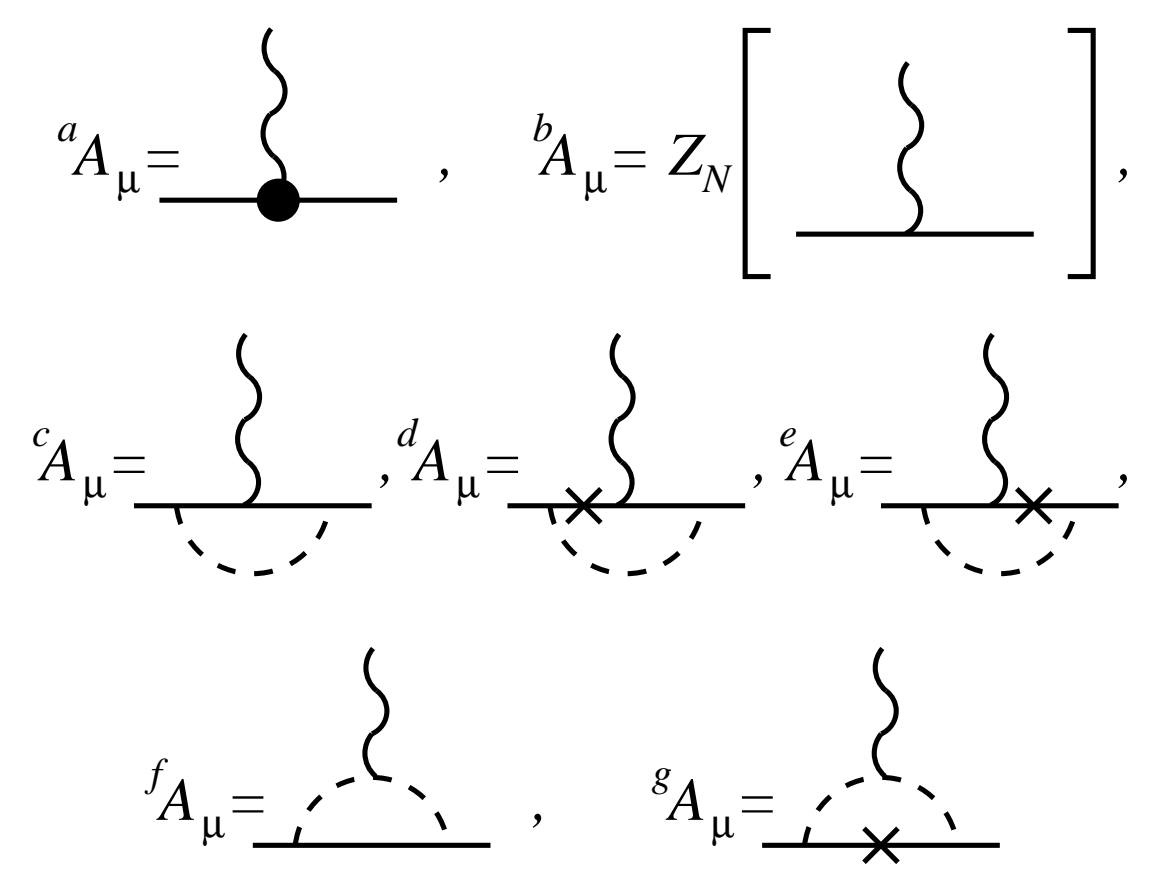

Figure 1: Contributions to the isospin-violating vector form factors of a nucleon at $O\left(p^{4}\right)$. A dashed line represents the sum over charged and neutral pions. The solid dot denotes an insertion from $\mathcal{L}_{\pi N}^{(3)}+\mathcal{L}_{\pi N}^{(4)}$, a cross denotes an insertion from $\mathcal{L}_{\pi N}^{(2)}$, and all other Feynman rules come from $\mathcal{L}_{\pi N}^{(1)}+\mathcal{L}_{\pi \pi}^{(2)}$.

The wave function renormalization constant for $\mathcal{L}_{\pi N}$ with the field redefinitions of Ref. [13] has been determined previously up to $O\left(p^{3}\right)$ [15, 16], but the present work will require an extension of the isospin-violating part to $O\left(p^{4}\right)$. The relevant diagrams are displayed in Fig. 2. Using dimensional regularization, the calculation of a diagram without an $O\left(p^{2}\right)$ insertion proceeds as follows:

$$
\begin{aligned}
{ }^{a} A_{Z}= & \mu^{4-d} \int \frac{\mathrm{d}^{d} \ell}{(2 \pi)^{d}}\left(\frac{i}{\ell^{2}-m_{\pi^{0}}^{2}+i \epsilon}\right)\left(-\frac{g_{A}}{F} S \cdot \ell\right)\left(\frac{i}{v \cdot(k-\ell)+i \epsilon}\right)\left(\frac{g_{A}}{F} S \cdot \ell\right) \\
= & \frac{-3 i g_{A}^{2}}{4(4 \pi F)^{2}}\left[v \cdot k\left(m_{\pi^{0}}^{2}-\frac{2}{3}(v \cdot k)^{2}\right)\left(\frac{2}{4-d}+1-\gamma+\ln (4 \pi)-\ln \frac{m_{\pi^{0}}^{2}}{\mu^{2}}\right)\right. \\
& \left.+\frac{2}{3} v \cdot k\left(m_{\pi^{0}}^{2}-(v \cdot k)^{2}\right)-\frac{4}{3}\left(m_{\pi^{0}}^{2}-(v \cdot k)^{2}\right)^{3 / 2}\left(\frac{\pi}{2}+\arcsin \frac{v \cdot k}{m_{\pi^{0}}}\right)\right] .
\end{aligned}
$$

Although this expression contains no explicit quark masses, it can still violate isospin by 


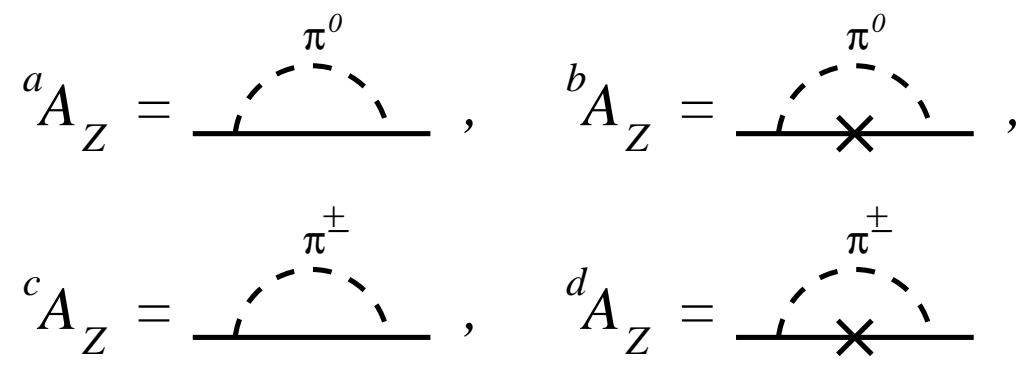

Figure 2: Contributions to the isospin-violating piece of the nucleon's wave function renormalization constant. A cross denotes an insertion from $\mathcal{L}_{\pi N}^{(2)}$, and all other Feynman rules come from $\mathcal{L}_{\pi N}^{(1)}+\mathcal{L}_{\pi \pi}^{(2)}$

virtue of the on-shell relation between $v \cdot k$ and the nucleon masses as given in Eq. (28). The wave function renormalization constant is defined to be the residue of the nucleon propagator at the on-shell point (multiplied by $i$ ), so Eq. (38) should be viewed as a function of $x \equiv v \cdot k-M_{N}+M_{0}$. The resulting contribution of this one diagram to the difference between proton and neutron wave function renormalization constants is

$$
\delta\left(Z_{p}-Z_{n}\right)=i \frac{\mathrm{d}}{\mathrm{d} x}\left[{ }^{a} A_{Z}(p)-{ }^{a} A_{Z}(n)\right]_{x=0}=-\frac{3}{2}\left(M_{n}-M_{p}\right) \frac{\pi g_{A}^{2} m_{\pi^{0}}}{(4 \pi F)^{2}}+O\left(M_{n}-M_{p}\right)^{2} .
$$

Similar contributions are made by the other diagrams in Fig. 2,

$$
\begin{aligned}
i \frac{\mathrm{d}}{\mathrm{d} x}\left[{ }^{b} A_{Z}(p)-{ }^{b} A_{Z}(n)\right]_{x=0} & =\frac{3}{2}\left(M_{n}-M_{p}\right) \frac{\pi g_{A}^{2} m_{\pi^{0}}}{(4 \pi F)^{2}}+O\left(M_{n}-M_{p}\right)^{2}, \\
i \frac{\mathrm{d}}{\mathrm{d} x}\left[{ }^{c} A_{Z}(p)-{ }^{c} A_{Z}(n)\right]_{x=0} & =-3\left(M_{n}-M_{p}\right) \frac{\pi g_{A}^{2} m_{\pi^{+}}}{(4 \pi F)^{2}}+O\left(M_{n}-M_{p}\right)^{2}, \\
i \frac{\mathrm{d}}{\mathrm{d} x}\left[{ }^{d} A_{Z}(p)-{ }^{d} A_{Z}(n)\right]_{x=0} & =-3\left(M_{n}-M_{p}\right) \frac{\pi g_{A}^{2} m_{\pi^{+}}}{(4 \pi F)^{2}}+O\left(M_{n}-M_{p}\right)^{2} .
\end{aligned}
$$

Adding these four contributions to the lower-order result of Eq. (33) yields

$$
Z_{p}-Z_{n}=\frac{M_{n}-M_{p}}{M_{0}}-6\left(M_{n}-M_{p}\right) \frac{\pi g_{A}^{2} m_{\pi^{+}}}{(4 \pi F)^{2}}
$$

The expressions for the matrix elements corresponding to the diagrams of Fig. 1] are now presented in the rest frame of the initial nucleon with $v_{\mu}=(1,0,0,0)$, on-shell external nucleons, and an isoscalar vector current with incoming momentum $q_{\mu}$ :

$$
{ }^{a} A_{\mu}^{(u+d)}(p)-{ }^{a} A_{\mu}^{(u+d)}(n)=\text { const } \times i\left(M_{n}-M_{p}\right) i \epsilon_{\mu \nu \rho \sigma} q^{\nu} v^{\rho} S^{\sigma},
$$




$$
\begin{aligned}
{ }^{b} A_{\mu}^{(u+d)}(p)-{ }^{b} A_{\mu}^{(u+d)}(n) & \left.=-i\left(M_{n}-M_{p}\right)\left[6 v_{\mu} \frac{\pi g_{A}^{2} m_{\pi^{+}}}{(4 \pi F)^{2}}-\frac{q_{\mu}}{2 M_{0}^{2}}-\frac{2 a_{7}}{M_{0}^{2}} i \epsilon_{\mu \nu \rho \sigma} q^{\nu} v^{\rho} S^{\sigma}\right] 4,5\right) \\
{ }^{c} A_{\mu}^{(u+d)}(p)-{ }^{c} A_{\mu}^{(u+d)}(n) & =\frac{3}{2} i v_{\mu}\left(M_{n}-M_{p}\right) \frac{\pi g_{A}^{2}}{(4 \pi F)^{2}}\left(2 m_{\pi^{+}}+m_{\pi^{0}}\right), \\
{ }^{d} A_{\mu}^{(u+d)}(p)-{ }^{d} A_{\mu}^{(u+d)}(n) & ={ }^{e} A_{\mu}^{(u+d)}(p)-{ }^{e} A_{\mu}^{(u+d)}(n) \\
& =\frac{3}{4} i v_{\mu}\left(M_{n}-M_{p}\right) \frac{\pi g_{A}^{2}}{(4 \pi F)^{2}}\left(2 m_{\pi^{+}}-m_{\pi^{0}}\right), \\
{ }^{f} A_{\mu}^{(u+d)}(p)-{ }^{f} A_{\mu}^{(u+d)}(n) & ={ }^{g} A_{\mu}^{(u+d)}(p)-{ }^{g} A_{\mu}^{(u+d)}(n)=0 .
\end{aligned}
$$

Their sum is

$$
A_{\mu}^{(u+d)}(p)-A_{\mu}^{(u+d)}(n)=i\left(M_{n}-M_{p}\right)\left[\frac{q_{\mu}}{2 M_{0}^{2}}+\text { const } \times i \epsilon_{\mu \nu \rho \sigma} q^{\nu} v^{\rho} S^{\sigma}\right] .
$$

The contribution from Eq. (32) has also been absorbed into the unspecified constant. According to Eqs. (19) and (20), the term containing $i \epsilon_{\mu \nu \rho \sigma} q^{\nu} v^{\rho} S^{\sigma}$ in Eq. (49) is the leading contribution to ${ }^{u+d} F_{2}^{p-n}\left(q^{2}\right)$. If there had been a term containing $v_{\mu}$, it would have been the leading contribution to ${ }^{u+d} F_{1}^{p-n}\left(q^{2}\right)$. As discussed in the previous section, ${ }^{u+d} F_{1}^{p-n}(0)=0$ is required by Noether's theorem, and this is explicitly verified by the exact cancellation of terms proportional to $v_{\mu}$ in Eqs. (44)-(48).

There is also a term in Eq. (49) that is proportional to $q_{\mu}$, and its origin is more subtle. Instead of contributing to the isospin violation of the form factors, this $q_{\mu}$ term is a consequence of the nucleon mass dependence in the currents themselves. Recall that Eqs. (19) and (20) are expressed in terms of the physical nucleon mass rather than the bare mass. Thus,

$$
\left[\bar{\psi}(\vec{p}) \gamma_{\mu} \psi(\vec{p})\right]_{p}-\left[\bar{\psi}(\vec{p}) \gamma_{\mu} \psi(\vec{p})\right]_{n}=\frac{\left(M_{n}-M_{p}\right)}{2 M_{0}^{2}} \bar{N}_{v}\left[q_{\mu}+2 i \epsilon_{\mu \nu \rho \sigma} q^{\nu} v^{\rho} S^{\sigma}+O\left(\frac{1}{M_{0}^{4}}\right)\right] N_{v} .
$$

This term containing $q_{\mu}$ is precisely the one shown in Eq. (49), while the term containing the antisymmetric tensor is absorbed into the unspecified constant in Eq. (49). Therefore, the $O\left(p^{4}\right)$ expressions for the " $u+d$ " vector form factors are

$$
\begin{aligned}
& { }^{u+d} F_{1}^{p-n}\left(q^{2}\right)=0, \\
& { }^{u+d} F_{2}^{p-n}\left(q^{2}\right)=\text { const } \equiv{ }^{u+d} \kappa^{p-n} .
\end{aligned}
$$

The form factors for an isovector ( $u-d$ ") vector current are less trivial because they receive a nonvanishing contribution from those diagrams in Fig. 1 where the vector current 
couples directly to a pion. Those are the diagrams which produce momentum dependence at $O\left(p^{4}\right)$. The complete set of " $u-d$ " contributions leads to

$$
\begin{aligned}
{ }^{u-d} F_{1}^{p+n}\left(q^{2}\right)= & -\frac{12 \pi g_{A}^{2}}{(4 \pi F)^{2}} m_{\pi^{+}}\left(M_{n}-M_{p}\right)\left[1-\frac{4}{3} \int_{0}^{1} \mathrm{~d} x \sqrt{1-x(1-x) \frac{q^{2}}{m_{\pi^{+}}^{2}}}\right. \\
& \left.+\frac{1}{3} \int_{0}^{1} \mathrm{~d} x\left(1-x(1-x) \frac{q^{2}}{m_{\pi^{+}}^{2}}\right)^{-1 / 2}\right], \\
{ }^{u-d} F_{2}^{p+n}\left(q^{2}\right)= & { }^{u-d} \kappa^{p+n}+\frac{16 g_{A}^{2} M_{N}}{(4 \pi F)^{2}}\left(M_{n}-M_{p}\right) \int_{0}^{1} \mathrm{~d} x \ln \left(1-x(1-x) \frac{q^{2}}{m_{\pi^{+}}^{2}}\right) .
\end{aligned}
$$

The numerical value of ${ }^{u-d} \kappa^{p+n}$ is not specified by chiral symmetry. It should be noted that Eq. (53) satisfies the constraint that ${ }^{u-d} F_{1}^{p+n}(0)=0$, which is a nontrivial check of the algebra since it results from a cancellation among the diagrams of Fig. 1.

Throughout this section, pion-loop diagrams have been evaluated while the effects of virtual photon loops were tacitly omitted. This omission can be justified by simple power-counting arguments. To begin, recall that the chiral Lagrangian is order-by-order renormalizable, and that any two-loop diagram cannot contribute before $O\left(p^{5}\right)$. (Photon loops have the same power counting as pion loops due to the association $O(e) \sim O(p)[1]$.) Up to $O\left(p^{4}\right)$ then, the only effects of virtual photons come from the addition of a single virtual photon to the simplest tree-level form factor diagram.

Such a one-photon-loop diagram could offer an $O\left(p^{3}\right)$ contribution to ${ }^{i} F_{1}^{j}\left(q^{2}\right)$. However, this form factor is required to vanish at $q^{2}=0$, so there must be an extra factor of $q^{2} / M_{0}^{2}$ at least, which then contributes at $O\left(p^{5}\right)$. This stands in contrast to the pion-loop diagrams which contain the extra mass scale $m_{\pi}$; the ratio $q^{2} / m_{\pi}^{2}$ does not lead to extra suppression in the HBChPT expansion.

A one-photon-loop diagram could also offer a contribution to ${ }^{i} F_{2}{ }^{j}\left(q^{2}\right)$, which begins at $O\left(p^{4}\right)$ rather than $O\left(p^{3}\right)$, due to the explicit factor of $q / M_{N}$ in the definition of these form factors, Eqs. (2) and (3). The contribution must be a simple constant because any $q^{2}$ dependence would require extra factors of $1 / M_{0}$ and would thus contribute at a higher order. The constant $O\left(p^{4}\right)$ contribution from virtual photons simply adjusts the numerical value of the unspecified parameters, ${ }^{i} \kappa^{j}$, in Eqs. (52) and (54).

The $O\left(p^{4}\right)$ isospin-violating " $u-d$ " form factors are plotted in Fig. 3 for $0<-q^{2}<$ $0.25 \mathrm{GeV}^{2}$, with ${ }^{u-d} \kappa^{p+n}$ set to zero and the numerical values of all other quantities set 


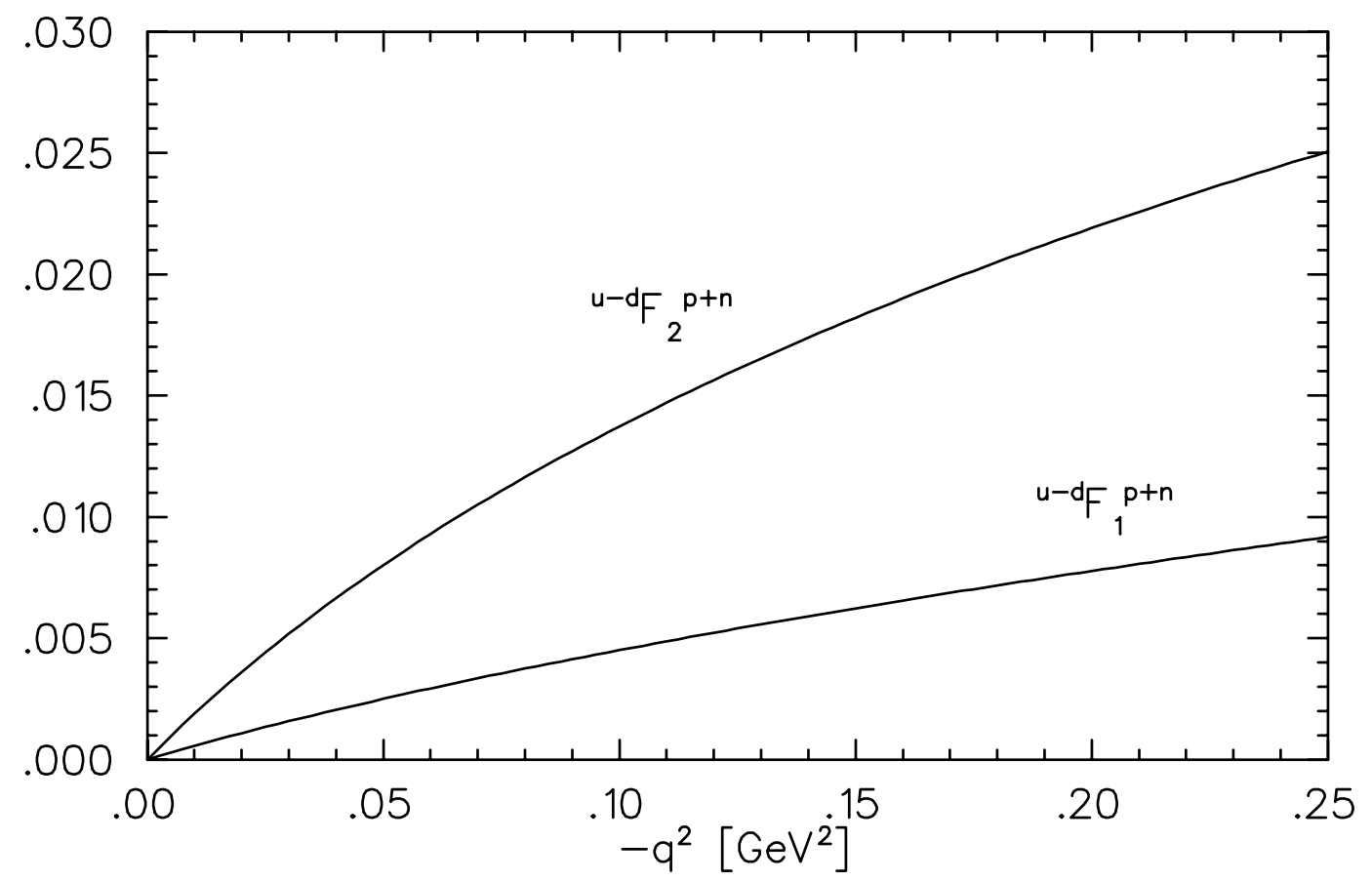

Figure 3: Leading-order HBChPT results for the isospin-violating isovector form factors. For this plot, the unspecified constant in ${ }^{u-d} F_{2}^{p+n}$ has been chosen such that the form factor vanishes at $q^{2}=0$.

to those of the Particle Data Group. [18] Uncertainties are not shown in Fig. 3. As will be discussed in the following section, the dominant uncertainty comes from the truncation of the HBChPT expansion at $O\left(p^{4}\right)$, and an estimate of this uncertainty will be obtained from the $O\left(p^{5}\right)$ calculation.

Independent of the value of ${ }^{u-d} \kappa^{p+n}$, Fig. 3 indicates that both " $u-d$ " form factors are monotonically increasing at leading order, with ${ }^{u-d} F_{2}^{p+n}$ increasing more quickly than ${ }^{u-d} F_{1}^{p+n}$.

According to Eqs. (5)-(7), it is the sum of these two form factors which is relevant to the proton's neutral weak magnetic form factor. However, ${ }^{i} F_{1}^{j}$ and ${ }^{i} F_{2}^{j}$ differ by an explicit power of $M_{N}$ due to their definition, Eqs. (2) and (3). Thus, the leading order 
result from $\mathrm{HBChPT}$ is

$$
G_{M}^{u, d}\left(q^{2}\right)={ }^{u+d} \kappa^{p-n}-{ }^{u-d} \kappa^{p+n}-\frac{16 g_{A}^{2} M_{N}}{(4 \pi F)^{2}}\left(M_{n}-M_{p}\right) \int_{0}^{1} \mathrm{~d} x \ln \left(1-x(1-x) \frac{q^{2}}{m_{\pi^{+}}^{2}}\right) .
$$

The $O\left(p^{4}\right)$ result for ${ }^{u-d} F_{1}^{p+n}$ is a next-to-leading-order correction to $G_{M}^{u, d}$, and it is reassuring to see from Fig. 3 that its $q^{2}$-dependence is smaller than the leading $q^{2}$-dependence.

The remaining next-to-leading-order effects come from an $O\left(p^{5}\right)$ calculation of ${ }^{i} F_{2}^{j}$, and are discussed in the following section.

\section{NEXT-TO-LEADING ORDER, $O\left(p^{5}\right)$}

The next-to-leading corrections for both the ${ }^{i} F_{1}^{j}$ and ${ }^{i} F_{2}^{j}$ isospin-violating form factors occur at $O\left(p^{5}\right)$ in HBChPT. The goal of the present section is to complete the next-toleading calculation of $G_{M}^{u, d} \cdot{ }^{i} F_{1}^{j}$ is itself a subleading contribution to $G_{M}^{u, d}$, so the $O\left(p^{4}\right)$ result of the previous section is a next-to-leading order effect. Therefore only ${ }^{i} F_{2}^{j}$ needs to be calculated at $O\left(p^{5}\right)$.

To construct the set of contributing Feynman diagrams, consider first those diagrams which contain no propagating pions (i.e. tree-level diagrams or diagrams containing any number of photon loops). The only available dimensionful parameters are the nucleon mass and momentum transfer, and it is easily concluded that such diagrams cannot contribute to the isospin-violating ${ }^{i} F_{2}^{j}$ at $O\left(p^{5}\right)$. Also, no contribution emerges from any diagram which contains both one photon loop and one pion loop.

A diagram containing two pion loops can only contribute at $O\left(p^{5}\right)$ if all Feynman rules come from $\mathcal{L}_{\pi N}^{(1)}+\mathcal{L}_{\pi \pi}^{(2)}$. However, no isospin violation is contained within $\mathcal{L}_{\pi N}^{(1)}$, and the $m_{\pi^{+}}-m_{\pi^{0}}$ mass difference does not affect the form factors of interest here, so only one-pion-loop diagrams can contribute.

The set of diagrams which do contribute includes ${ }^{b} A_{\mu},{ }^{f} A_{\mu}$ and ${ }^{g} A_{\mu}$ in Fig. 11, plus the diagrams of Fig. 4 . Although $\mathcal{L}_{\pi N}^{(3)}$ contains about 40 parameters that are unconstrained by chiral symmetry and could in principle appear within $O\left(p^{5}\right)$ loop diagrams, none of them contribute to this calculation. Dimensional arguments do not permit a tree-level $O\left(p^{5}\right)$ counterterm for ${ }^{i} F_{2}^{j}$, (essentially because the small expansion parameters without uncontracted Lorentz indices tend to come in pairs at tree-level, such as $m_{\pi}^{2}$, $e^{2}$, or $q^{2}$, but $O\left(p^{5}\right)$ would require an odd power), so the total loop calculation must be finite. 


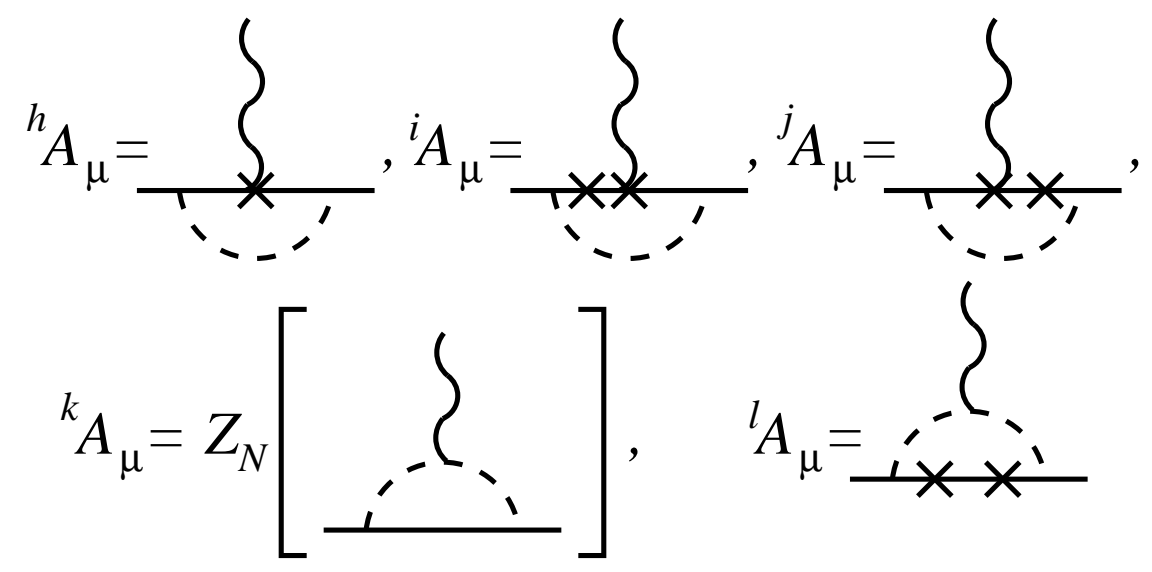

Figure 4: Contributions to the isospin-violating ${ }^{i} F_{2}^{j}$ form factors that begin at $O\left(p^{5}\right)$. A dashed line represents the sum over charged and neutral pions. A cross denotes an insertion from $\mathcal{L}_{\pi N}^{(2)}$, and all other Feynman rules come from $\mathcal{L}_{\pi N}^{(1)}+\mathcal{L}_{\pi \pi}^{(2)}$.

Summing the $O\left(p^{5}\right)$ contributions and adding them to the $O\left(p^{4}\right)$ results from the preceding section produces the full isospin-violating contribution to the proton's neutral weak magnetic moment, as computed within HBChPT (without explicit $\Delta$ (1232) fields),

$$
\begin{aligned}
G_{M}^{u, d}\left(q^{2}\right)= & { }^{u+d} \kappa^{p-n}-{ }^{u-d} \kappa^{p+n}-\frac{16 g_{A}^{2} M_{N}}{(4 \pi F)^{2}}\left(M_{n}-M_{p}\right) \int_{0}^{1} \mathrm{~d} x \ln \left(1-x(1-x) \frac{q^{2}}{m_{\pi^{+}}^{2}}\right) \\
& -\left(M_{n}-M_{p}\right) \frac{24 \pi g_{A}^{2} m_{\pi^{+}}}{(4 \pi F)^{2}}\left[\frac{\mu_{p}+\mu_{n}}{\mu_{N}}-\frac{1}{2}-\frac{4}{3} \int_{0}^{1} \mathrm{~d} x \sqrt{1-x(1-x) \frac{q^{2}}{m_{\pi^{+}}^{2}}}\right. \\
& \left.+\frac{1}{6} \int_{0}^{1} \mathrm{~d} x\left(1-x(1-x) \frac{q^{2}}{m_{\pi^{+}}^{2}}\right)^{-1 / 2}\right] .
\end{aligned}
$$

The parameters $a_{6}$ and $a_{7}$ have been re-expressed as the nucleon magnetic moments via Eq. (23),

$$
\begin{aligned}
& a_{6}=\frac{\mu_{p}-\mu_{n}}{4 \mu_{N}} \approx 1.176, \\
& a_{7}=\frac{\mu_{p}+\mu_{n}}{2 \mu_{N}} \approx 0.440,
\end{aligned}
$$

plus higher-order corrections that are not needed for the present work. $\mu_{N}$ is the nuclear magneton. 


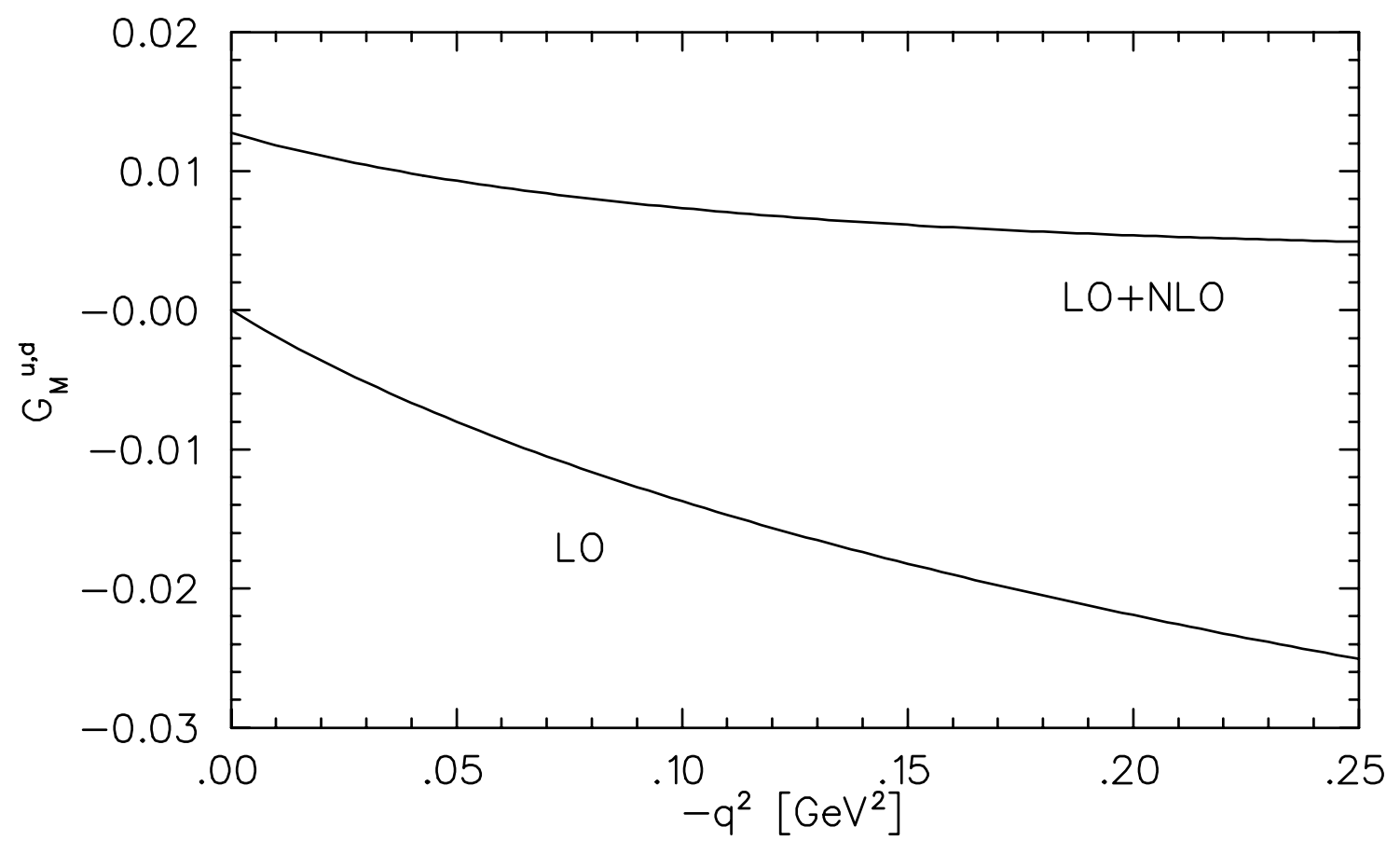

Figure 5: The leading order (LO) and next-to-leading order (NLO) HBChPT results for the isospin-violating contribution to the proton's neutral weak magnetic moment. For this plot, the unspecified constant at LO has been chosen such that the form factor vanishes at $q^{2}=0$. There are no unspecified parameters in the NLO contribution.

Fig. 5 contains a plot of $G_{M}^{u, d}\left(q^{2}\right)$ versus $q^{2}$ at leading order (LO) and next-to-leading order (NLO), with the only unspecified quantity ${ }^{u+d} \kappa^{p-n}-{ }^{u-d} \kappa^{p+n}$ set to zero. The NLO effects serve to soften the $q^{2}$-dependence of the form factor. Fig. 5 indicates that the NLO corrections to $G_{M}^{u, d}\left(q^{2}\right)$ total roughly 0.01 at $q^{2}=0$, and grow to about 0.02 near $q^{2}=0.1 \mathrm{GeV}^{2}$.

To determine how well the HBChPT expansion is working for this observable, it is useful to consider the derivative of the form factor at $q^{2}=0$,

$$
\frac{\mathrm{d}}{\mathrm{d}\left(-q^{2}\right)} G_{M}^{u, d}(0)=-\frac{8 g_{A}^{2} M_{N}\left(M_{n}-M_{p}\right)}{3 m_{\pi^{+}}^{2}(4 \pi F)^{2}}\left(1-\frac{9 \pi m_{\pi^{+}}}{8 M_{N}}\right) .
$$

Thus the ratio of magnitudes of the $\mathrm{NLO} / \mathrm{LO}$ contributions is $9 \pi m_{\pi^{+}} / 8 M_{N} \approx 1 / 2$. Taking this as representative, the uncertainty from the neglect of NNLO effects could be roughly 
half of the NLO contribution. These uncertainties will be discussed further in section 6 .

\section{INCLUDING THE DELTA RESONANCE}

Conspicuous by its absence from the preceding discussion is the $\Delta(1232)$ isomultiplet. The effects of an infinitely-heavy $\Delta(1232)$ are accommodated within the numerical values of the HBChPT parameters, but the rather small $\Delta-N$ mass difference observed in nature raises the possibility of substantial corrections to the $M_{\Delta} \rightarrow \infty$ limit.

The incorporation of an explicit $\Delta(1232)$ field into HBChPT was initiated by Jenkins and Manohar [9] and has been employed by various authors. [19, 20] In this section, the formalism developed by Hemmert, Holstein and Kambor in Ref. [20] is used to calculate the leading $\Delta(1232)$ contributions to the isospin-violating ${ }^{i} F_{1}^{j}$ and ${ }^{i} F_{2}^{j}$ form factors.

As is familiar from relativistic approaches to spin-3/2 field theory, the Lagrangian contains a vector-spinor and one then employs a projection operator to isolate the spin$3 / 2$ piece. For example, the lowest-order propagator in " $d$ " spacetime dimensions is

$$
\left(\frac{-i}{v \cdot k-\Delta+i \epsilon}\right)\left[g_{\mu \nu}-v_{\mu} v_{\nu}+\left(\frac{4}{d-1}\right) S_{\mu} S_{\nu}\right],
$$

where the incoming 4-momentum is $M_{\Delta, 0} v_{\mu}+k_{\mu}, M_{\Delta, 0}$ is the lowest-order $\Delta(1232)$ mass, and

$$
\Delta \equiv M_{\Delta, 0}-M_{0} .
$$

As expected, the leading contributions of the $\Delta(1232)$ to the isospin-violating form factors appear at $O\left(p^{4}\right)$. The relevant diagrams are displayed in Fig. 6, and the terms required from the leading-order Lagrangian are

$$
\begin{aligned}
\delta \mathcal{L}_{\pi \Delta}^{(1)} & =-\bar{T}_{i}^{\mu}\left[i v \cdot D^{i j}-\delta^{i j} \Delta\right] T_{\mu j}, \\
\delta \mathcal{L}_{\pi N \Delta}^{(1)} & =g_{\pi N \Delta}\left[\bar{T}_{i}^{\mu} \omega_{\mu}^{i} N+\bar{N} \omega_{\mu}^{i \dagger} T_{i}^{\mu}\right],
\end{aligned}
$$

where $T_{\mu}^{i}$ is the vector-spinor, $\omega_{\mu}^{i}$ is defined by

$$
\omega_{\mu}^{i}=\frac{1}{2} \operatorname{Tr}\left(\tau^{i} u_{\mu}\right)
$$

( $\tau^{i}$ is a Pauli matrix in isospin space) and the covariant derivative is

$$
D_{\mu}^{i j}=\delta^{i j}\left(\partial_{\mu}+\Gamma_{\mu}-i v_{\mu}^{(s)}\right)-2 i \epsilon^{i j k} \Gamma_{\mu}^{k} .
$$




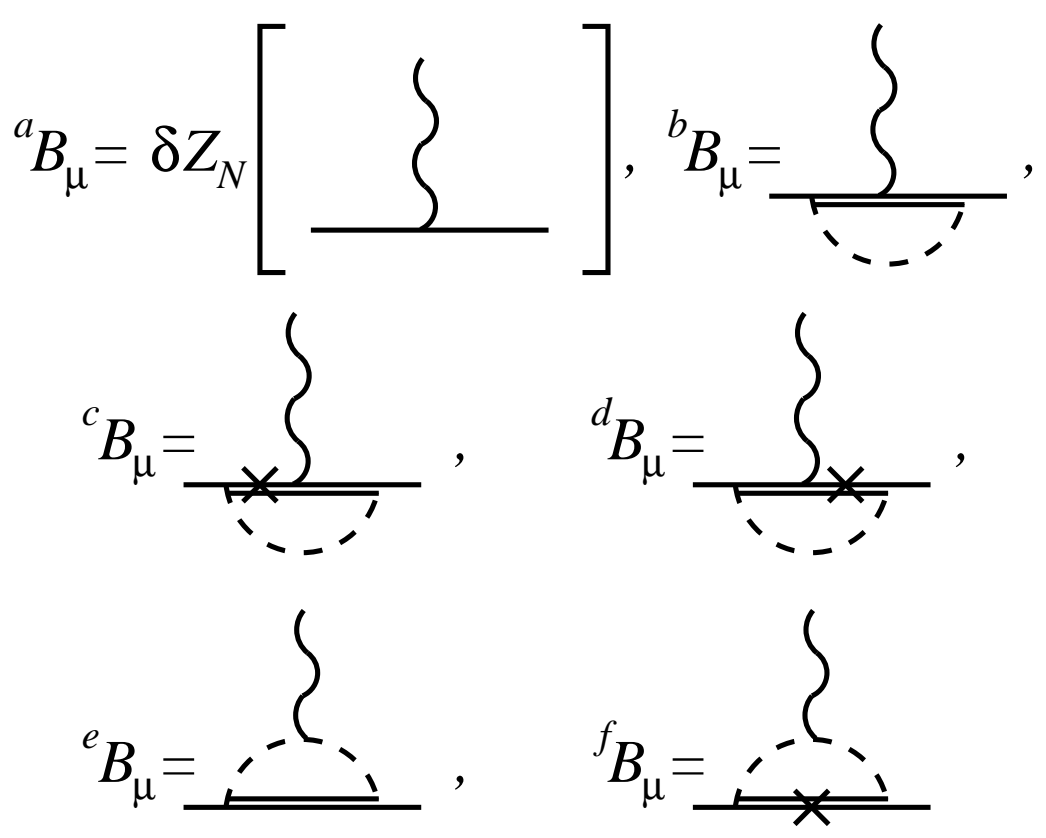

Figure 6: Contributions of the $\Delta(1232)$ to the isospin-violating vector form factors of a nucleon at $O\left(p^{4}\right)$. A dashed line represents the sum over charged and neutral pions, and a double line represents the $\Delta(1232)$. A cross denotes an insertion from $\mathcal{L}_{\pi \Delta}^{(2)}$, and all other Feynman rules come from $\mathcal{L}_{\pi N}^{(1)}+\mathcal{L}_{\pi N \Delta}^{(1)}+\mathcal{L}_{\pi \pi}^{(2)}$.

The only insertions from $\mathcal{L}^{(2)}$ that contribute are the $\Delta(1232)$ mass corrections, which arise from electromagnetic as well as strong interaction effects.

The authors of Ref. [20] chose not to perform the $\Delta(1232)$ field transformation that would have removed "equation of motion" terms from $\mathcal{L}^{(2)}$. None of the calculations in this section depend upon whether or not the transformation is performed.

The leading contributions of the $\Delta(1232)$ to nucleon wave function renormalization are shown diagrammatically in Fig. 17. When their contribution is added to the non- $\Delta(1232)$ result of Eq. (43), the full isospin-violation due to wave function renormalization is found to be

$$
Z_{p}-Z_{n}=\frac{M_{n}-M_{p}}{M_{0}}-6\left(M_{n}-M_{p}\right) \frac{\pi g_{A}^{2} m_{\pi^{+}}}{(4 \pi F)^{2}}
$$



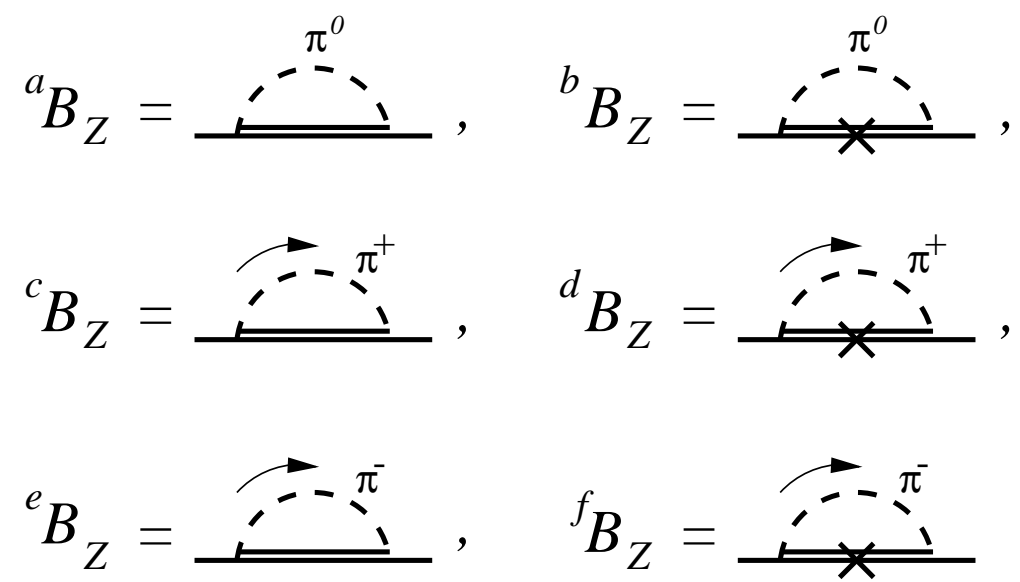

Figure 7: Contributions of the $\Delta(1232)$ to the isospin-violating piece of the nucleon's wave function renormalization constant. A double line represents the $\Delta(1232)$. A cross denotes an insertion from $\mathcal{L}_{\pi \Delta}^{(2)}$, and all other Feynman rules come from $\mathcal{L}_{\pi \Delta}^{(1)}+\mathcal{L}_{\pi N \Delta}^{(1)}+\mathcal{L}_{\pi \pi}^{(2)}$.

$$
\begin{aligned}
& -\frac{8}{3}\left[4\left(M_{n}-M_{p}\right)+\left(M_{\Delta^{0}}-M_{\Delta^{+}}\right)-3\left(M_{\Delta^{-}}-M_{\Delta^{++}}\right)\right] R\left(m_{\pi^{+}}^{2}, 0\right) \\
& -\frac{16}{3}\left[M_{n}-M_{p}-\left(M_{\Delta^{0}}-M_{\Delta^{+}}\right)\right] R\left(m_{\pi^{0}}^{2}, 0\right) .
\end{aligned}
$$

where

$$
\begin{aligned}
R\left(m^{2}, q^{2}\right) \equiv & \frac{g_{\pi N \Delta}^{2}}{(4 \pi F)^{2}}\left[\Delta\left(\frac{1}{\epsilon}-\gamma+\ln (4 \pi)-\ln \frac{m^{2}}{\mu^{2}}\right)-\Delta \int_{0}^{1} \mathrm{~d} x \ln \left(1-x(1-x) \frac{q^{2}}{m^{2}}\right)\right. \\
& -\frac{\Delta}{3} \int_{0}^{1} \mathrm{~d} x\left(\frac{x(1-x) q^{2}}{\Delta^{2}-m^{2}+x(1-x) q^{2}}\right) \\
& -\int_{0}^{1} \mathrm{~d} x\left(\frac{2 \Delta^{2}-m^{2}+x(1-x) q^{2}}{\sqrt{\Delta^{2}-m^{2}+x(1-x) q^{2}}}-\frac{x(1-x) q^{2}\left[m^{2}-x(1-x) q^{2}\right]}{3\left[\Delta^{2}-m^{2}+x(1-x) q^{2}\right]^{3 / 2}}\right) \\
& \left.\times \ln \left(\frac{\Delta}{\sqrt{m^{2}-x(1-x) q^{2}}}+\sqrt{\frac{\Delta^{2}}{m^{2}-x(1-x) q^{2}}-1}\right)\right] .
\end{aligned}
$$

The set of $O\left(p^{4}\right)$ diagrams, contained in Fig. 6, produces the following contributions to the isospin-violating form factors:

$$
\delta\left[{ }^{u+d} F_{1}^{p-n}\left(q^{2}\right)\right]=\delta\left[{ }^{u+d} F_{2}^{p-n}\left(q^{2}\right)\right]=0
$$




$$
\begin{aligned}
\delta\left[{ }^{u-d} F_{1}^{p+n}\left(q^{2}\right)\right]= & -\frac{16}{3}\left[2\left(M_{n}-M_{p}\right)-\left(M_{\Delta^{0}}-M_{\Delta^{+}}\right)-3\left(M_{\Delta^{-}}-M_{\Delta^{++}}\right)\right] \\
& \times\left[R\left(m_{\pi^{+}}^{2}, q^{2}\right)-R\left(m_{\pi^{+}}^{2}, 0\right)\right] \\
\delta\left[{ }^{u-d} F_{2}^{p+n}\left(q^{2}\right)\right]= & \frac{8 g_{\pi N \Delta}^{2}}{9(4 \pi F)^{2}} M_{N}\left[2\left(M_{n}-M_{p}\right)-\left(M_{\Delta^{0}}-M_{\Delta^{+}}\right)-3\left(M_{\Delta^{-}}-M_{\Delta^{++}}\right)\right] \\
& \times\left[\operatorname{const}+\int_{0}^{1} \mathrm{~d} x \ln \left(1-x(1-x) \frac{q^{2}}{m_{\pi^{+}}^{2}}\right)\right. \\
& +2 \int_{0}^{1} \mathrm{~d} x \frac{\Delta}{\sqrt{\Delta^{2}-m_{\pi^{+}}^{2}+x(1-x) q^{2}}} \\
& \left.\times \ln \left(\frac{\Delta}{\sqrt{m_{\pi^{+}}^{2}-x(1-x) q^{2}}}+\sqrt{\frac{\Delta^{2}}{m_{\pi^{+}}^{2}-x(1-x) q^{2}}-1}\right)\right]
\end{aligned}
$$

Notice that the total contribution made by the $\Delta(1232)$ loop graphs to each of the " $u+d$ " form factors exactly vanishes. For the isovector ( " $u-d$ ") current, the various contributions to ${ }^{u-d} F_{1}^{p+n}$ add in such a way that all divergences cancel and the familiar constraint, ${ }^{u-d} F_{1}^{p+n}(0)=0$ is satisfied. As expected, the contribution to ${ }^{u-d} F_{2}^{p+n}$ is not finite, and the full Lagrangian contains an $O\left(p^{4}\right)$ counterterm which absorbs this divergence.

Plotted in Fig. 8 are the leading results, i.e. the $O\left(p^{4}\right)$ results, for the two " $u-d$ " form factors with and without the explicit $\Delta(1232)$ contribution, and with the unspecified constant subtracted from ${ }^{u-d} F_{2}^{p+n}$. The effects of the $\Delta(1232)$ are significantly smaller than the NLO corrections of Fig. 5. The value $g_{\pi N \Delta}=1.05$, recommended in Ref. [20], has been used along with the following mass differences:

$$
\begin{aligned}
\Delta & =0.293 \mathrm{GeV} \\
M_{\Delta^{0}}-M_{\Delta^{+}} & =\frac{1}{3}\left(M_{\Delta^{-}}-M_{\Delta^{++}}\right)=.0013 \mathrm{GeV}
\end{aligned}
$$

There are sizable experimental uncertainties on these inputs, but no reasonable choices can make the $\Delta(1232)$ contribution to $G_{M}^{u, d}\left(q^{2}\right)$ grow larger than the NLO correction of Fig. 5.

\section{DISCUSSION}

The proton's neutral weak magnetic form factor $G_{M}^{p, Z}$ is of great interest, both experimentally and theoretically, because it is sensitive to strangeness within the nucleon. 


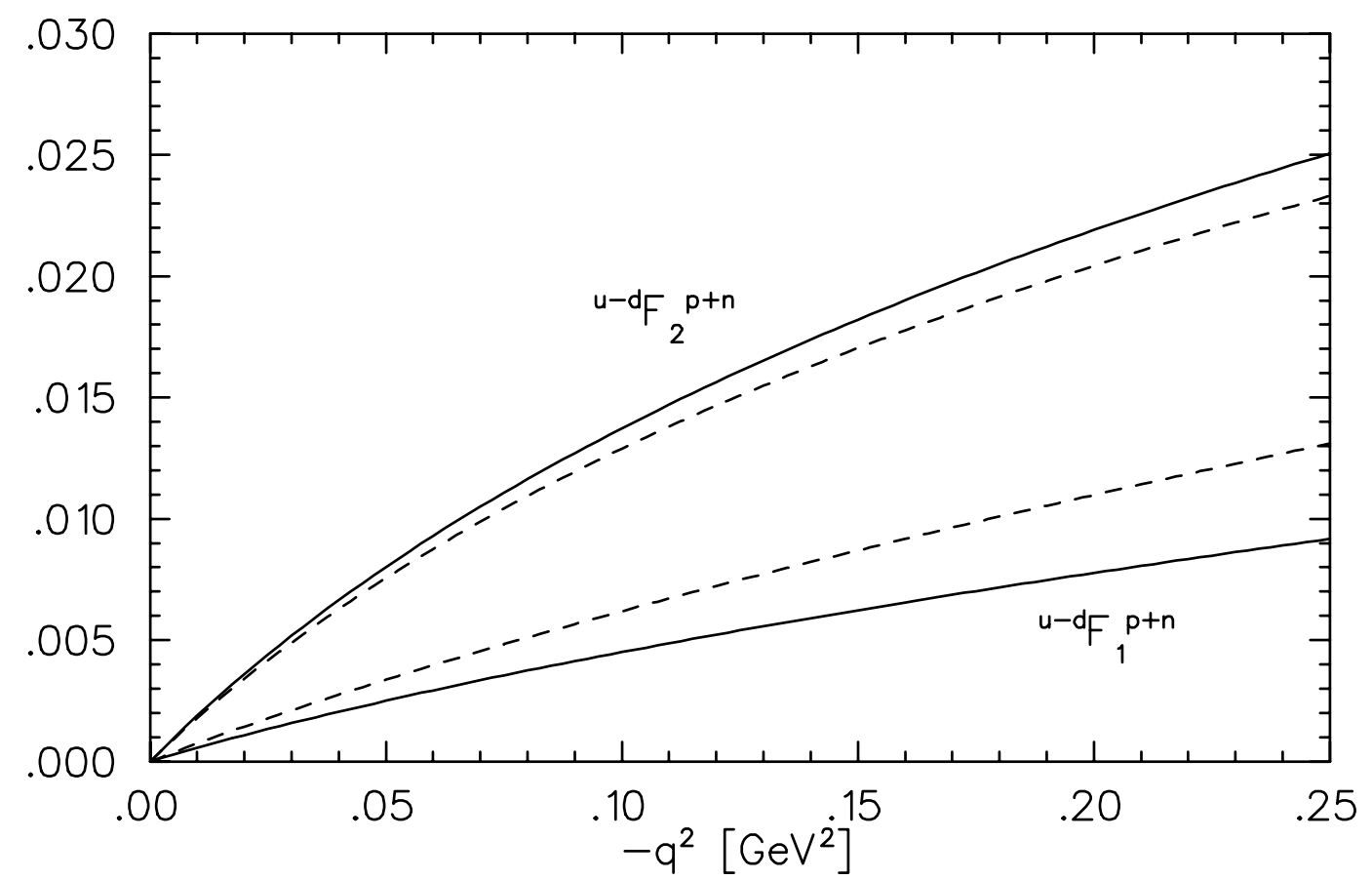

Figure 8: Leading-order HBChPT results for the isospin-violating isovector form factors without (solid lines) and with (dashed lines) the explicit $\Delta(1232)$ isomultiplet. For this plot, the unspecified constant in ${ }^{u-d} F_{2}^{p+n}$ has been chosen such that the form factor vanishes at $q^{2}=0$.

Experiments actually measure the sum of strangeness and isospin-violating contributions (labeled $G_{M}^{s}$ and $G_{M}^{u, d}$ respectively), so it is advantageous to understand the effects of isospin violation as thoroughly as possible.

In this work, heavy baryon chiral perturbation theory (HBChPT) has been used to study isospin violation in the absence of strange quarks. HBChPT is an expansion in momenta that are small compared to the chiral scale, $\Lambda_{\chi} \sim m_{\rho} \sim M_{N} \sim 4 \pi F_{\pi}$. Virtual photons can also been included according to the usual $\alpha_{\mathrm{QED}}$ expansion.

First, $G_{M}^{u, d}\left(q^{2}\right)$ was calculated at leading order (LO) and next-to-leading order (NLO) for a single nucleon surrounded by a cloud of pions and photons. The photon cloud was found to contain no momentum dependence at this order, in contrast to the pion cloud. The ratio of NLO/LO contributions was used as an estimator of the systematic uncertainty of the HBChPT expansion. Then the contribution of the $\Delta(1232)$ isomultiplet 
was evaluated and found to be negligible in comparison with the systematic uncertainty.

The final expression is given by Eq. (56) in terms of a single unspecified parameter, ${ }^{u+d} \kappa^{p-n}-{ }^{u-d} \kappa^{p+n}$. This parameter contains physics of two types: a low-energy contribution from the pion cloud, and a higher-energy contribution which is unconstrained by HBChPT.

The pion-cloud contribution to the unspecified parameter is easily determined by redoing the HBChPT calculation with a momentum cutoff, $\lambda$, instead of dimensional regularization. This cutoff represents the separation scale between the "low-energy" and "higher-energy" regions. In principle, the choice of $\lambda$ does not affect $G_{M}^{u, d}\left(q^{2}\right)$ since only the sum of low-energy and higher-energy pieces is relevant, but in HBChPT the higherenergy piece is undetermined so it is preferable to make a physical choice for $\lambda$.

Clearly $\lambda$ cannot be larger than $\Lambda_{\chi}$, since HBChPT fails above this scale. In a series of recent papers [21], Donoghue, Holstein and Borasoy have argued for an HBChPT cutoff that is not too far above $1 \mathrm{fm}^{-1} \approx 200 \mathrm{MeV}$, corresponding to the measured size of a baryon. Above this approximate scale the substructure of a nucleon can begin to be relevant, but is not accurately represented in $\mathrm{HBChPT}$.

The only divergence in the non- $\Delta(1232)$ piece of $G_{M}^{u, d}\left(q^{2}\right)$ comes from a single integral which appears in diagrams ${ }^{f} A_{\mu}$ and ${ }^{g} A_{\mu}$ of Figure 11. When dimensional regularization is replaced by a simple cutoff for the momentum integral, the following relationship is obtained:

$$
\left(\frac{2}{4-d}-\gamma+\ln (4 \pi)-\ln \frac{m_{\pi^{+}}^{2}}{\mu^{2}}\right) \rightarrow\left(\ln \frac{\lambda^{2}}{m_{\pi^{+}}^{2}}-\frac{3}{2}\right)
$$

Choosing $\lambda=400 \mathrm{MeV}$, the low-energy contribution of the pion cloud is found to be

$$
\left[{ }^{u+d} \kappa^{p-n}-{ }^{u-d} \kappa^{p+n}\right]_{\text {pion cloud }}=0.014
$$

Notice that the dependence on $\lambda$ is only logarithmic, so the result is not overly sensitive to the chosen numerical value of the cutoff. The $\Delta(1232)$ contribution also contains a logarithmic dependence on $\lambda$, but the resulting pion-cloud contribution is negligible in comparison to the uncertainties coming from the HBChPT expansion (recall section 5).

The remaining contribution to the $G_{M}^{u, d}$ counterterm is the "higher-energy" contribution. It is unspecified in HBChPT by definition, and a precise numerical prediction is therefore beyond the scope of this work. If the HBChPT expansion is to be well-behaved, 
then the higher-energy contributions must respect the established power counting. Perhaps the most obvious examples of higher-energy physics are the $\rho$ and $\omega$ vector mesons. Simple power counting estimates for the tree-level vector meson dominance diagrams indicate that their leading contribution resembles

$$
\left[G_{M}^{u, d}\right]_{\rho, \omega} \propto \frac{M_{N}\left(M_{n}-M_{p}\right)}{m_{\rho, \omega}^{2}}
$$

which is $O\left(p^{4}\right)$, as required by $\mathrm{HBChPT}$.

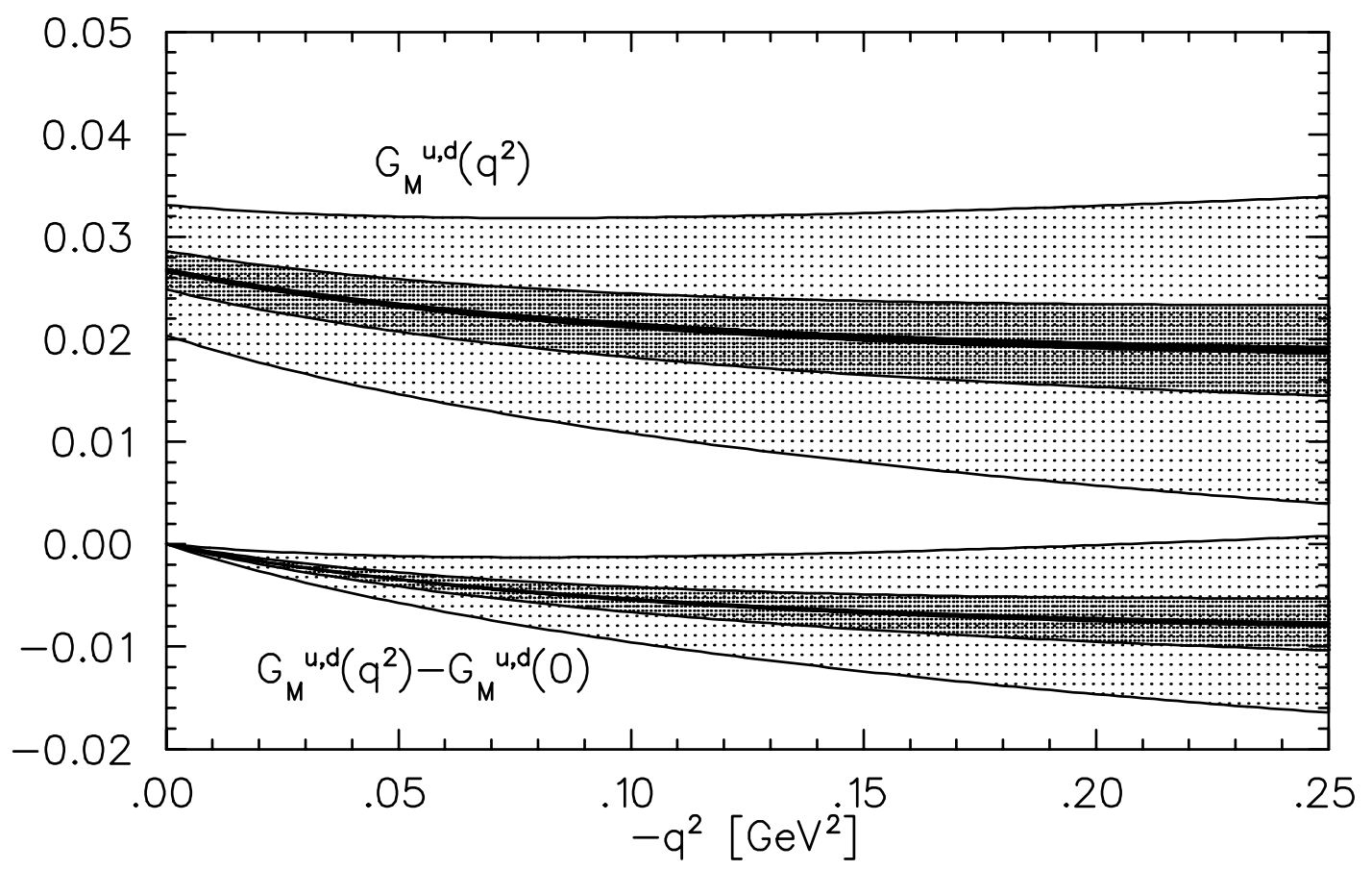

Figure 9: The HBChPT prediction for the isospin-violating pion-cloud contribution to the proton's neutral weak magnetic form factor. The two solid lines represent the central value with and without a subtraction at $q^{2}=0$, and a pair of uncertainty estimates are provided for each solid line according to Eq. (76). The logarithmic divergence is cut off at $400 \mathrm{MeV}$, as discussed in the text.

The main conclusion of this work is summarized by Fig. 9, which shows the full contribution of the pion cloud to $G_{M}^{u, d}\left(q^{2}\right)$, for $0<-q^{2}<0.25 \mathrm{GeV}^{2}$, up to next-toleading order in the HBChPT expansion. To aid a discussion of $q^{2}$-dependence, the same 
quantity is shown after $G_{M}^{u, d}(0)$ has been subtracted. In each case, a pair of uncertainty bands is shown, representing two estimates of the error associated with the neglect of NNLO contributions,

$$
|\mathrm{NNLO}| \sim\left\{\begin{array}{l}
\frac{1}{2}|\mathrm{NLO}|, \text { wide band } \\
\frac{m_{\pi}}{M_{N}}|\mathrm{NLO}|, \text { narrow band }
\end{array} .\right.
$$

The wide-band error estimate purports that the ratio of $1 / 2$, taken from $|\mathrm{NLO}| /|\mathrm{LO}|$ in Eq. (59) for the derivative of $G_{M}^{u, d}$, might be a reasonable indicator of the NNLO uncertainty. The narrow-band error estimate employs a generic HBChPT expansion parameter.

Fig. 9 indicates that the $q^{2}$-dependence, as determined by $G_{M}^{u, d}\left(q^{2}\right)-G_{M}^{u, d}(0)$, occurs on the scale of a few times 0.001, but is typically less than 0.01. This result is easily understood: HBChPT demands that the momentum dependence of this isospin-violating form factor is proportional to $\left(M_{n}-M_{p}\right) / M_{N} \approx 0.001$. Recall that no contributions from $m_{\pi^{+}}-m_{\pi^{0}}$ or any of the HBChPT parameters were found.

However, Fig. 9 also indicates that the contributions of the pion cloud to $G_{M}^{u, d}(0)$ are on the scale of a few times 0.01 . The origin of isospin violation is still solely $\left(M_{n}-M_{p}\right) / M_{N} \approx$ 0.001 , but the numerical coefficients are larger than those for the $q^{2}$-dependence.

It is interesting to compare the results of Fig. 9 to the findings of other authors. Dmitrašinović and Pollock have used a nonrelativistic constituent quark model to find[5]

$$
\left[{ }^{u+d} G_{M}^{p-n}(0)\right]_{\mathrm{DmiP}}=\left[{ }^{u-d} G_{M}^{p+n}(0)\right]_{\mathrm{DmiP}} \approx 0.008 \quad \Rightarrow \quad\left[G_{M}^{u, d}(0)\right]_{\mathrm{DmiP}}=0 .
$$

A vanishing total result at $q^{2}=0$ is also obtained by Miller, who has studied a family of three nonrelativistic constituent quark models. [7] As well, his work suggests that the $q^{2}$-dependence is very mild:

$$
\left[G_{M}^{u, d}(0)-G_{M}^{u, d}\left(-0.25 \mathrm{GeV}^{2}\right)\right]_{\mathrm{Mil}}<0.001
$$

Capstick and Robson have work in progress that employs a relativized constituent quark model. [6] Using a light-cone meson-baryon fluctuation model, Ma has reported the following allowed range[8]:

$$
\left[G_{M}^{u, d}(0)\right]_{\mathrm{Ma}}=0.006 \rightarrow 0.088 .
$$

In light of the uncertainties assigned to HBChPT, there is no essential disagreement between the present work and any of these models. Certainly the tendency of nonrelativistic quark models to prefer $G_{M}^{u, d}(0)=0$ is not obtained from the pion cloud in HBChPT, but recall that the effect of higher-energy physics remains unspecified in HBChPT. 
In conclusion, the effects of isospin violation on the proton's neutral weak magnetic form factor have been studied up to next-to-leading order in heavy baryon chiral perturbation theory. The momentum dependence contains no free parameters, and comes solely from the neutron-proton mass difference despite the large number of parameters in the Lagrangian. Normalization of the isospin-violating contribution at $q^{2}=0$ is not specified by chiral symmetry, but the pion-cloud effects can be extracted and their contribution is roughly 0.02 nuclear magnetons.

\section{ACKNOWLEDGMENTS}

We are grateful to José Goity for a critical reading of the manuscript. This research was supported in part by the Natural Sciences and Engineering Research Council of Canada. R.L. also acknowledges support from the U.S. Department of Energy, contract DE-AC0584ER40150.

\section{References}

[1] B. Mueller et. al., Phys. Rev. Lett. 78, 3824 (1997).

[2] Jefferson Lab experiments E91-004 (E. J. Beise, spokesperson); E91-010 (P. Souder and J. Finn, spokespersons); E91-017 (D. Beck, spokesperson).

[3] M. J. Musolf and B. R. Holstein, Phys. Lett. B242, 461 (1990).

[4] For a review, see M. J. Musolf, T. W. Donnelly, J. Dubach, S. J. Pollock, S. Kowalski and E. J. Beise, Phys. Rep. 239, 1 (1994).

[5] V. Dmitrašinović and S. J. Pollock, Phys. Rev. C52, 1061 (1995).

[6] S. Capstick and D. Robson, Florida State preprint FSU-SCRI-97-96, nucl-th/9708054 (1997). Numerical results in the first version of this preprint are expected to change (S. Capstick, private communication).

[7] G. A. Miller, Phys. Rev. C57, 1492 (1998). 
[8] B.-Q. Ma, Phys. Lett. B408, 387 (1997).

[9] E. Jenkins and A. V. Manohar, Phys. Lett. B255, 558 (1991).

[10] For two useful reviews, see V. Bernard, N. Kaiser and U.-G. Meissner, Int J. Mod. Phys. E4, 193 (1995); U.-G. Meissner, to appear in the proceedings of the 12th Annual HUGS at CEBAF, hep-ph/9711365 (1997).

[11] U.-G. Meissner and S. Steininger, Phys. Lett. B419, 403 (1998).

[12] T. R. Hemmert, U.-G. Meissner and S. Steininger, Jülich preprint FZJ-IKP(TH)1998-12, hep-ph/9806226 (1998).

[13] G. Ecker and M. Mojžiš, Phys. Lett. B365, 312 (1996).

[14] N. Fettes, U.-G. Meissner and S. Steininger, Jülich preprint FZJ-IKP(TH)-1998-03, hep-ph/9803266 (1998).

[15] H. W. Fearing, R. Lewis, N. Mobed and S. Scherer, Phys. Rev. D56, 1783 (1997).

[16] G. Ecker and M. Mojžiš, Phys. Lett. B410, 266 (1997).

[17] U.-G. Meissner, G. Müller and S. Steininger, Jülich preprint FZJ-IKP(TH)-1998-08, hep-ph/9809446 (1998).

[18] Particle Data Group, C. Caso et. al., Eur. Phys. J. C3, 1 (1998).

[19] Some examples include M. N. Butler, M. J. Savage and R. P. Springer, Phys. Rev. D49, 3459 (1994) and references therein; A. Abd El-Hady, J. Tandean and G. Valencia, Iowa State University Report No. ISU-HET-98-2, hep-ph/9808322 (1998) and references therein.

[20] T. R. Hemmert, B. R. Holstein and J. Kambor, Phys. Lett. B395, 89 (1997); TRIUMF Report No. TRI-PP-97-21, hep-ph/9712496 (1997).

[21] J. F. Donoghue and B. R. Holstein, hep-ph/9803312 (1998); J. F. Donoghue, B. R. Holstein and B. Borasoy, hep-ph/9804281 (1998); B. Borasoy, hep-ph/9807453 (1998). 